\title{
The Jacobian Consistency of a One-Parametric Class of Smoothing Functions for SOCCP
}

\author{
Xiaoni Chi, ${ }^{1,2}$ Zhongping Wan, $^{1}$ and Zijun Hao ${ }^{1,3}$ \\ ${ }^{1}$ School of Mathematics and Statistics, Wuhan University, Wuhan 430072, China \\ ${ }^{2}$ School of Mathematics and Computing Science, Guilin University of Electronic Technology, Guilin 541004, China \\ ${ }^{3}$ School of Information and Calculating Science, North University for Ethnics, Yinchuan 750021, China
}

Correspondence should be addressed to Zhongping Wan; mathwanzhp@whu.edu.cn

Received 22 February 2013; Accepted 21 May 2013

Academic Editor: Ming Mei

Copyright (C) 2013 Xiaoni Chi et al. This is an open access article distributed under the Creative Commons Attribution License, which permits unrestricted use, distribution, and reproduction in any medium, provided the original work is properly cited.

\begin{abstract}
Second-order cone (SOC) complementarity functions and their smoothing functions have been much studied in the solution of second-order cone complementarity problems (SOCCP). In this paper, we study the directional derivative and B-subdifferential of the one-parametric class of SOC complementarity functions, propose its smoothing function, and derive the computable formula for the Jacobian of the smoothing function. Based on these results, we prove the Jacobian consistency of the one-parametric class of smoothing functions, which will play an important role for achieving the rapid convergence of smoothing methods. Moreover, we estimate the distance between the subgradient of the one-parametric class of the SOC complementarity functions and the gradient of its smoothing function, which will help to adjust a parameter appropriately in smoothing methods.
\end{abstract}

\section{Introduction}

The second-order cone complementarity problem (SOCCP) [1] is to find $(x, y, \zeta) \in R^{n} \times R^{n} \times R^{l}$ such that

$$
x \in K, y \in K, x^{T} y=0, \quad F(x, y, \zeta)=0,
$$

where $F: R^{n} \times R^{n} \times R^{l} \rightarrow R^{n} \times R^{l}$ is a continuously differentiable mapping, $K \subset R^{n}$ is the Cartesian product of second-order cones (SOC), that is, $K=K^{n_{1}} \times K^{n_{2}} \times \cdots \times K^{n_{m}}$ with $n=n_{1}+n_{2}+\cdots+n_{m}$ and the $n_{i}$-dimensional SOC defined by

$$
K^{n_{i}}=\left\{x^{i}=\left(x_{1}^{i} ; x_{2}^{i}\right) \in R \times R^{n_{i}-1}: x_{1}^{i}-\left\|x_{2}^{i}\right\| \geq 0\right\} .
$$

Without loss of generality, we may assume that $m=1$ and $K=K^{n}$ in the following analysis, since our analysis can be easily extended to the general case.

The SOCCP contains a wide class of problems, such as nonlinear complementarity problems [2], second-order cone programming $[1,3,4]$, and has a variety of engineering and management applications, such as filter design, antenna array weight design, truss design, and grasping force optimization in robotics $[5,6]$.
Recently, great attention has been paid to smoothing methods, partially due to their superior theoretical and numerical performances [7-10]. Smoothing methods usually reformulate the SOCCP as a system of equations by using smoothing functions of SOC complementarity functions $[10,11]$. The smoothing parameter involved in smoothing functions may be treated as a variable [9] or a parameter with an appropriate parameter control [7]. In the latter case, the Jacobian consistency plays a key role for achieving a rapid convergence of Newton the methods or the Newton-like methods. Hayashi et al. [7] propose a combined smoothing and regularized method for monotone SOCCP and show its global and quadratic convergence based on the Jacobian consistency of the smoothing natural residual function. Ogasawara and Narushima [12] show the Jacobian consistency of a smoothed Fischer-Burmeister (FB) function. Chen et al. [13] present a smoothing function of a generalized FB function in the context of nonlinear complementarity programming and study some of its favorable properties, including the Jacobian consistency property. Based on the results, they [13] propose a smoothing algorithm for the mixed complementarity problem, which is shown to possess 
global convergence and local superlinear (or quadratic) convergence.

In this paper, we are concerned with the one-parametric class of SOC complementarity functions $\phi_{\tau}: R^{n} \rightarrow R^{n}$ defined by [14]

$$
\begin{aligned}
\phi_{\tau}(x, y):= & x+y \\
& -\sqrt{x^{2}+y^{2}+(\tau-2)(x \circ y)},
\end{aligned}
$$

where $\tau \in(0,4)$ is an arbitrary but fixed parameter. When $\tau=2, \phi_{\tau}$ reduces to the vector-valued Fischer-Burmeister function given by

$$
\phi_{\mathrm{FB}}(x, y):=x+y-\sqrt{x^{2}+y^{2}}
$$

and as $\tau \rightarrow 0$, it becomes a multiple of the following vectorvalued residual function:

$$
\phi_{\mathrm{NR}}(x, y):=x-\Pi_{K^{n}}(x-y),
$$

where $\Pi_{K^{n}}(\cdot)$ denotes the metric projection on the secondorder cone $K^{n}$. Thus, the one-parametric class of vectorvalued functions (3) cover two popular second-order cone complementarity functions.

In this paper, we aim to show the Jacobian consistency of smoothing functions of the one-parametric class of SOC complementarity functions, which will play an important role for achieving the rapid convergence of smoothing methods. Moreover, we estimate the distance between the subgradient of the one-parametric class of the SOC complementarity functions and the gradient of the smoothing functions, which will help to adjust a parameter appropriately in smoothing methods.

The organization of this paper is as follows. In Section 2, we review some preliminaries including the Euclidean Jordan algebra associated with SOC and subdifferentials. In Section 3, we derive the computable formula for the Jacobian of the one-parametric class of smoothing functions in the SOCCP. In Section 4, we prove the Jacobian consistency of the one-parametric class of smoothing functions and estimate the distance between the gradient of the smoothing functions and the subgradient of the one-parametric class of the SOC complementarity functions. In Section 5, we study the directional derivative and $B$-subdifferential of the oneparametric class of SOC complementarity functions and then present an alternative way to prove the Jacobian consistency of the one-parametric class of smoothing functions. Finally, we close this paper with some conclusions in Section 6.

In what follows, we denote the nonnegative orthant of $R$ by $R_{+}$. We use the symbol $\|\cdot\|$ to denote the Euclidean norm defined by $\|x\|:=\sqrt{x^{T} x}$ for a vector $x$ or the corresponding induced matrix norm. For simplicity, we often use $x=$ $\left(x_{1} ; x_{2}\right)$ for the column vector $x=\left(x_{1}, x_{2}^{T}\right)^{T}$. For the SOC $K^{n}$, int $K^{n}$ and bd $K^{n}$ mean the topological interior and the boundary of $K^{n}$, respectively. For a given set $S \subset R^{m \times n}$, conv $S$ denotes the convex hull of $S$ in $R^{m \times n}$, and $\operatorname{dist}(X, S)$ denotes $\inf \{\|X-Y\|: Y \in S\}$ for a matrix $X \in R^{m \times n}$.

\section{Preliminaries}

In this section, we recall some concepts and results, which include the Euclidean Jordan algebra $[3,15]$ associated with the SOC $K^{n}$ and subdifferentials [16].

First, we recall the Euclidean Jordan algebra associated with the SOC and some useful definitions. The Euclidean Jordan algebra for the SOC $K^{n}$ is the algebra defined by

$$
x \circ y=\left(x^{T} y ; x_{1} y_{2}+y_{1} x_{2}\right), \quad \forall x, y \in R^{n},
$$

with $e=(1,0, \ldots, 0) \in R^{n}$ being its unit element. Given an element $x=\left(x_{1} ; x_{2}\right) \in R \times R^{n-1}$, we define

$$
L(x)=\left(\begin{array}{ll}
x_{1} & x_{2}^{T} \\
x_{2} & x_{1} I
\end{array}\right),
$$

where $I$ represents the $(n-1) \times(n-1)$ identity matrix. It is easy to verify that $x \circ y=L(x) y$ for any $y \in R^{n}$. Moreover, $L(x)$ is symmetric positive definite (and hence invertible) if and only if $x \in$ int $K^{n}$.

Now, we give the spectral factorization of vectors in $R^{n}$ associated with the SOC $K^{n}$. Let $x=\left(x_{1} ; x_{2}\right) \in R \times R^{n-1}$. Then $x$ can be decomposed as

$$
x=\lambda_{1} u^{(1)}+\lambda_{2} u^{(2)},
$$

where $\lambda_{1}, \lambda_{2}$, and $u^{(1)}, u^{(2)}$ are the spectral values and the associated spectral vectors of $x$ given by

$$
\begin{gathered}
\lambda_{i}=x_{1}+(-1)^{i}\left\|x_{2}\right\|, \\
u^{(i)}= \begin{cases}\frac{1}{2}\left(1,(-1)^{i} \frac{x_{2}}{\left\|x_{2}\right\|}\right) & \text { if } x_{2} \neq 0, \\
\frac{1}{2}\left(1,(-1)^{i} \omega\right) & \text { otherwise, }\end{cases}
\end{gathered}
$$

for $i=1,2$, with any $\omega \in R^{n-1}$ such that $\|\omega\|=1$. By the spectral factorization, a scalar function can be extended to a function for the SOC. For any $x \in R^{n}$, we define

$$
x^{2}=\lambda_{1}^{2} u^{(1)}+\lambda_{2}^{2} u^{(2)} .
$$

Since both eigenvalues of any $x \in K^{n}$ are nonnegative, we define

$$
\sqrt{x}=\sqrt{\lambda_{1}} u^{(1)}+\sqrt{\lambda_{2}} u^{(2)}
$$

For any $x=\left(x_{1} ; x_{2}\right) \in R \times R^{n-1}$, we define [12] $x^{\prime}=$ $\left(x_{1} ;-x_{2}\right)$. Obviously, $x^{\prime \prime}=x,(x+y)^{\prime}=x^{\prime}+y^{\prime}$, and $(c x)^{\prime}=$ $c x^{\prime}$ for any $c \in R$. Moreover, $x \circ x^{\prime}=x_{1}^{2}-\left\|x_{2}\right\|^{2}=0$ if $x \in \mathrm{bd} K^{n}$.

Let $G: R^{m} \rightarrow R^{n}$ be a locally Lipschitzian function. Then, $G$ is differentiable almost everywhere by Rademacher's theorem [17]. The Bouligand- (B-) subdifferential and the Clarke subdifferential of $G$ at $z$ are defined by

$$
\begin{gathered}
\partial G_{B}(z):=\left\{\lim _{\bar{z} \rightarrow z} \nabla G(\widehat{z}): \widehat{z} \in D_{G}\right\}, \\
\partial G(z)=\operatorname{conv} \partial G_{B}(z),
\end{gathered}
$$


respectively, where $D_{G}$ denotes the set of points at which $G$ is differentiable. It is obvious that $\partial G(z)=\{\nabla G(z)\}$ if $G$ is continuously differentiable at $z$.

By using the concepts of subdifferentials, we give the definition of the Jacobian consistency, which was first introduced by Chen et al. [16], which is a concept relating the generalized Jacobian of a nonsmooth function with the Jacobian of a smoothing function [7].

Definition 1 (see [16]). Let $G: R^{m} \rightarrow R^{n}$ be a locally Lipschitzian function. Let $G_{\varepsilon}: R^{m} \rightarrow R^{n}$ be a continuously differentiable function for any $\varepsilon>0$ such that $\lim _{\varepsilon \downarrow 0} G_{\varepsilon}(z)=G(z)$ for any $z \in R^{m}$. We say that $G_{\varepsilon}$ satisfies the Jacobian consistency property if for any $z \in$ $R^{m}, \lim _{\mathcal{\varepsilon} \downarrow 0} \operatorname{dist}\left(\nabla G_{\varepsilon}(z), \partial G(z)\right)=0$.

It should be noted that the "inf" appearing in the definition of $\operatorname{dist}\left(\nabla G_{\varepsilon}(z), \partial G(z)\right)$ can be replaced by "min," since the set $\partial G(z)$ is compact at all $z \in R^{m}$ [17].

\section{Smoothing Function}

In this section, we propose a smoothing function of the one-parametric class of SOC complementarity functions and derive the computable formula for its Jacobian.

Since the one-parametric class of SOC complementarity functions $\phi_{\tau}$ defined by (3) is nonsmooth, we consider the function $\phi_{\tau, \varepsilon}$ defined by

$$
\begin{aligned}
\phi_{\tau, \varepsilon}(x, y):= & x+y \\
& -\sqrt{x^{2}+y^{2}+(\tau-2)(x \circ y)+2 \varepsilon^{2} e},
\end{aligned}
$$

where the smoothing parameter $\varepsilon \in R$.

Definition 2 (see [7]). For a nondifferentiable function $h$ : $R^{m} \rightarrow R^{n}$, one considers a function $h_{\varepsilon}: R^{m} \rightarrow R^{n}$ with a parameter $\varepsilon>0$ that has the following properties:

(i) $h_{\varepsilon}$ is differentiable for any $\varepsilon>0$;

(ii) $\lim _{\varepsilon \downarrow 0} h_{\varepsilon}(x)=h(x)$ for any $x \in R^{m}$.

Such a function $h_{\varepsilon}$ is called a smoothing function of $h$.

In the following, we will show that the function $\phi_{\tau, \varepsilon}$ given by (13) is a smoothing function of $\phi_{\tau}$. Thus, we can solve a family of smoothing subproblems $\phi_{\tau, \varepsilon}(x, y)=0$ for $\varepsilon>0$ and obtain a solution of $\phi_{\tau}(x, y)=0$ by letting $\varepsilon \downarrow 0$.

For convenience, we give some notations. For any $x=$ $\left(x_{1} ; x_{2}\right), y=\left(y_{1} ; y_{2}\right) \in R \times R^{n-1}$, and any $\varepsilon \in R$, we define the mapping $z^{\varepsilon}: R^{2 n} \rightarrow R \times R^{n-1}$ by

$$
\begin{aligned}
z^{\varepsilon} & =\left(z_{1}^{\varepsilon} ; z_{2}^{\varepsilon}\right) \\
& =z^{\varepsilon}(x, y):=x^{2}+y^{2}+(\tau-2)(x \circ y)+2 \varepsilon^{2} e,
\end{aligned}
$$

and drop the subscript for simplicity for $\varepsilon=0$, and thus,

$$
\begin{aligned}
z & =\left(z_{1} ; z_{2}\right) \\
& =z(x, y):=x^{2}+y^{2}+(\tau-2)(x \circ y) .
\end{aligned}
$$

By direct calculations, we obtain

$$
\begin{aligned}
z_{1}^{\varepsilon}= & \|x\|^{2}+\|y\|^{2} \\
& +(\tau-2) x^{T} y+2 \varepsilon^{2}=2 \varepsilon^{2}+z_{1}, \\
z_{2}^{\varepsilon}= & 2 x_{1} x_{2}+2 y_{1} y_{2} \\
& +(\tau-2)\left(x_{1} y_{2}+y_{1} x_{2}\right)=z_{2},
\end{aligned}
$$

and therefore $z^{\varepsilon}=\left(z_{1}^{\varepsilon} ; z_{2}\right)$. Then, the spectral factorization of $z^{\varepsilon}$ is

$$
z^{\varepsilon}=\lambda_{1}\left(z^{\varepsilon}\right) u_{1}(z)+\lambda_{2}\left(z^{\varepsilon}\right) u_{2}(z)
$$

where $\lambda_{1}\left(z^{\varepsilon}\right), \lambda_{2}\left(z^{\varepsilon}\right)$, and $u_{1}(z), u_{2}(z)$ are the spectral values and the associated spectral vectors of $z^{\varepsilon}$ given by

$$
\begin{aligned}
& \lambda_{i}\left(z^{\varepsilon}\right) \\
& =2 \varepsilon^{2}+\|x\|^{2} \\
& +\|y\|^{2}+(\tau-2) x^{T} y \\
& +(-1)^{i} \| 2 x_{1} x_{2}+2 y_{1} y_{2} \\
& +(\tau-2)\left(x_{1} y_{2}+y_{1} x_{2}\right) \|, \\
& u_{i}(z)=\frac{1}{2}\left(1 ;(-1)^{i} \bar{z}_{2}\right),
\end{aligned}
$$

for $i=1,2$. Here,

$$
\begin{aligned}
\bar{z}_{2}:= & \frac{z_{2}}{\left\|z_{2}\right\|} \\
& =\frac{2 x_{1} x_{2}+2 y_{1} y_{2}+(\tau-2)\left(x_{1} y_{2}+y_{1} x_{2}\right)}{\left\|2 x_{1} x_{2}+2 y_{1} y_{2}+(\tau-2)\left(x_{1} y_{2}+y_{1} x_{2}\right)\right\|},
\end{aligned}
$$

if $z_{2} \neq 0$, and otherwise, $\bar{z}_{2}$ is any vector in $R^{n-1}$ such that $\left\|\bar{z}_{2}\right\|=1$.

For any $(x, y) \in R^{n} \times R^{n}$, it is not difficult to verify that

$$
\begin{aligned}
z^{\varepsilon} & =x^{2}+y^{2}+(\tau-2)(x \circ y)+2 \varepsilon^{2} e \\
& =\left(x+\frac{\tau-2}{2} y\right)^{2}+\frac{\tau(4-\tau)}{4} y^{2}+2 \varepsilon^{2} e \\
& =\left(y+\frac{\tau-2}{2} x\right)^{2}+\frac{\tau(4-\tau)}{4} x^{2}+2 \varepsilon^{2} e \in \operatorname{int} K^{n},
\end{aligned}
$$

for any $\varepsilon \neq 0$, and

$$
\begin{aligned}
z & =x^{2}+y^{2}+(\tau-2)(x \circ y) \\
& =\left(x+\frac{\tau-2}{2} y\right)^{2}+\frac{\tau(4-\tau)}{4} y^{2} \\
& =\left(y+\frac{\tau-2}{2} x\right)^{2}+\frac{\tau(4-\tau)}{4} x^{2} \in K^{n} .
\end{aligned}
$$


Therefore, for any $x=\left(x_{1} ; x_{2}\right), y=\left(y_{1} ; y_{2}\right) \in R \times R^{n-1}$, and any $\varepsilon \in R$, we can also define

$$
\begin{aligned}
w^{\varepsilon} & =\left(w_{1}^{\varepsilon} ; w_{2}^{\varepsilon}\right) \\
& =w^{\varepsilon}(x, y) \\
& :=\sqrt{x^{2}+y^{2}+(\tau-2)(x \circ y)+2 \varepsilon^{2} e}
\end{aligned}
$$

and for $\varepsilon=0$,

$$
\begin{aligned}
w & =\left(w_{1} ; w_{2}\right) \\
& =w(x, y) \\
& :=\sqrt{x^{2}+y^{2}+(\tau-2)(x \circ y)} .
\end{aligned}
$$

The spectral factorization of $w^{\varepsilon}$ and $w$ is given by, respectively,

$$
\begin{gathered}
w^{\varepsilon}=\sqrt{\lambda_{1}\left(z^{\varepsilon}\right)} u_{1}(z)+\sqrt{\lambda_{2}\left(z^{\varepsilon}\right)} u_{2}(z) \\
w=\sqrt{\lambda_{1}(z)} u_{1}(z)+\sqrt{\lambda_{2}(z)} u_{2}(z)
\end{gathered}
$$

By (22), we can partition $R^{2 n}$ as $R^{2 n}=S_{1} \cup S_{2} \cup\{(0,0)\}$, where

$$
\begin{aligned}
S_{1} & :=\left\{(x, y) \in R^{2 n}: z \in \text { int } K^{n}\right\} \\
& =\left\{(x, y) \in R^{2 n}: \lambda_{2}(z) \geq \lambda_{1}(z)>0\right\}, \\
S_{2} & :=\left\{(x, y) \in R^{2 n}: z \in \operatorname{bd} K^{n} \backslash\{0\}\right\} \\
& =\left\{(x, y) \in R^{2 n}: 2 z_{1}=\lambda_{2}(z)>\lambda_{1}(z)=0\right\} .
\end{aligned}
$$

Theorem 3. For any $(x, y) \in R^{n} \times R^{n}$ and $\varepsilon \neq 0$, let $\phi_{\tau}$ and $\phi_{\tau, \varepsilon}$ be, respectively, defined by (3) and (13). Then, the following results hold.

(i) The function $\phi_{\tau, \varepsilon}$ is continuously differentiable everywhere, and its Jacobian is given by

$$
\begin{aligned}
& \nabla \phi_{\tau, \varepsilon}(x, y) \\
& =\left(\begin{array}{c}
I-L\left(x+\frac{\tau-2}{2} y\right) L^{-1}\left(w^{\varepsilon}\right) \\
I-L\left(y+\frac{\tau-2}{2} x\right) L^{-1}\left(w^{\varepsilon}\right)
\end{array}\right),
\end{aligned}
$$

where $L^{-1}\left(w^{\varepsilon}\right)=\left(1 / \sqrt{z_{1}^{\varepsilon}}\right) I$ if $z_{2}=0$, and otherwise,

$$
\begin{aligned}
L^{-1}\left(w^{\varepsilon}\right) & =L_{1}\left(z^{\varepsilon}\right)+L_{2}\left(z^{\varepsilon}\right) \\
& =\left(\begin{array}{cc}
b_{\varepsilon} & c_{\varepsilon} \bar{z}_{2}^{T} \\
c_{\varepsilon} \bar{z}_{2} & a_{\varepsilon} I+\left(b_{\varepsilon}-a_{\varepsilon}\right) \bar{z}_{2} \bar{z}_{2}^{T}
\end{array}\right),
\end{aligned}
$$

with

$$
\begin{gathered}
L_{1}\left(z^{\varepsilon}\right)=\frac{1}{2 \sqrt{\lambda_{1}\left(z^{\varepsilon}\right)}}\left(\begin{array}{cc}
1 & -\bar{z}_{2}^{T} \\
-\bar{z}_{2} & \bar{z}_{2} \bar{z}_{2}^{T}
\end{array}\right), \\
L_{2}\left(z^{\varepsilon}\right)= \\
\frac{1}{2 \sqrt{\lambda_{2}\left(z^{\varepsilon}\right)}}\left(\begin{array}{cc}
1 & \bar{z}_{2}^{T} \\
\bar{z}_{2} & \bar{z}_{2} \bar{z}_{2}^{T}
\end{array}\right) \\
+a_{\varepsilon}\left(\begin{array}{cc}
0 & 0^{T} \\
0 & I-\bar{z}_{2} \bar{z}_{2}^{T}
\end{array}\right)
\end{gathered}
$$

$$
\begin{gathered}
a_{\varepsilon}=\frac{2}{\sqrt{\lambda_{1}\left(z^{\varepsilon}\right)}+\sqrt{\lambda_{2}\left(z^{\varepsilon}\right)}}, \\
b_{\varepsilon}=\frac{1}{2}\left(\frac{1}{\sqrt{\lambda_{1}\left(z^{\varepsilon}\right)}}+\frac{1}{\sqrt{\lambda_{2}\left(z^{\varepsilon}\right)}}\right), \\
c_{\varepsilon}=\frac{1}{2}\left(\frac{1}{\sqrt{\lambda_{2}\left(z^{\varepsilon}\right)}}-\frac{1}{\sqrt{\lambda_{1}\left(z^{\varepsilon}\right)}}\right) .
\end{gathered}
$$

(ii) For any $(x, y) \in R^{n} \times R^{n}, \lim _{\varepsilon \downarrow 0} \phi_{\tau, \varepsilon}(x, y)=\phi_{\tau}(x, y)$. Thus, $\phi_{\tau, \varepsilon}$ is a smoothing function of $\phi_{\tau}$.

Proof. (i) For any $(x, y) \in R^{n} \times R^{n}$ and any $\varepsilon \neq 0$, it follows from Corollary 5.4 in [1], the chain rule for differentiation, and (21) that formula in (27) holds. Formula (28) is due to Proposition 5.2 and its proof in [1].

(ii) Fix any $x=\left(x_{1} ; x_{2}\right), y=\left(y_{1} ; y_{2}\right) \in R \times R^{n-1}$. For any $\varepsilon>0$, it follows from the spectral factorization of $z$ and $z^{\varepsilon}$ that

$$
\begin{aligned}
\phi_{\tau}(x, y)= & x+y \\
& -\left(\sqrt{\lambda_{1}(z)} u_{1}(z)+\sqrt{\lambda_{2}(z)} u_{2}(z)\right), \\
\phi_{\tau, \mathcal{\varepsilon}}(x, y)= & x+y \\
& -\left(\sqrt{\lambda_{1}\left(z^{\varepsilon}\right)} u_{1}(z)+\sqrt{\lambda_{2}\left(z^{\varepsilon}\right)} u_{2}(z)\right),
\end{aligned}
$$

where

$$
\begin{aligned}
\lambda_{i}(z)= & \|x\|^{2}+\|y\|^{2}+(\tau-2) x^{T} y \\
& +(-1)^{i} \| 2 x_{1} x_{2}+2 y_{1} y_{2} \\
& +(\tau-2)\left(x_{1} y_{2}+y_{1} x_{2}\right) \|,
\end{aligned}
$$

and $\lambda_{i}\left(z^{\varepsilon}\right)$ and $u_{i}(z)$ are, respectively, given by (18) and (19) for $i=1,2$. It is obvious that $\lambda_{i}\left(z^{\varepsilon}\right)=\lambda_{i}(z)+2 \varepsilon^{2}$ for $i=1,2$. Then,

$$
\begin{aligned}
& \lim _{\varepsilon \downarrow 0}\left(\sqrt{\lambda_{1}\left(z^{\varepsilon}\right)} u_{1}(z)+\sqrt{\lambda_{2}\left(z^{\varepsilon}\right)} u_{2}(z)\right) \\
& \quad=\lim _{\varepsilon \downarrow 0}\left(\sqrt{\lambda_{1}(z)+2 \varepsilon^{2}} u_{1}(z)+\sqrt{\lambda_{2}(z)+2 \varepsilon^{2}} u_{2}(z)\right) \\
& \quad=\sqrt{\lambda_{1}(z)} u_{1}(z)+\sqrt{\lambda_{2}(z)} u_{2}(z)
\end{aligned}
$$

and hence, $\lim _{\varepsilon \downarrow 0} \phi_{\tau, \varepsilon}(x, y)=\phi_{\tau}(x, y)$. Therefore, it follows from (i) and Definition 2 that $\phi_{\tau, \varepsilon}$ is a smoothing function of $\phi_{\tau}$. 
Next, we give some properties of $\phi_{\tau}$ [14], which will be used in the subsequent analysis.

Lemma 4. For any $x=\left(x_{1} ; x_{2}\right), y=\left(y_{1} ; y_{2}\right) \in R \times R^{n-1}$, let $z=\left(z_{1} ; z_{2}\right)=x^{2}+y^{2}+(\tau-2)(x \circ y) \in b d K^{n}$. Then one has

$$
\begin{gathered}
\left(x+\frac{\tau-2}{2} y\right) \circ\left(x+\frac{\tau-2}{2} y\right)^{\prime}=0, \quad y \circ y^{\prime}=0, \\
\left(x+\frac{\tau-2}{2} y\right) \circ z^{\prime}=0, \quad y \circ z^{\prime}=0, \\
\left(x+\frac{\tau-2}{2} y\right) \circ y^{\prime}=0, \\
\left(y+\frac{\tau-2}{2} x\right) \circ x^{\prime}=0, \\
\left(y+\frac{\tau-2}{2} x\right) \circ\left(y+\frac{\tau-2}{2} x\right)^{\prime}=0, \quad x \circ x^{\prime}=0, \\
\left(y+\frac{\tau-2}{2} x\right) \circ z^{\prime}=0, \quad x \circ z^{\prime}=0, \\
\left(x+\frac{\tau-2}{2} y\right) \circ\left(y+\frac{\tau-2}{2} x\right)^{\prime}=0, \quad x \circ y^{\prime}=0 .
\end{gathered}
$$

Moreover, the following equivalence holds:

$$
\begin{aligned}
z_{1}=0 & \Longleftrightarrow z_{2}=0 \Longleftrightarrow z=0 \\
& \Longleftrightarrow x_{1}=y_{1}=0 \Longleftrightarrow x_{2}=y_{2}=0 \\
& \Longleftrightarrow(x, y)=(0,0) .
\end{aligned}
$$

Proof. From Lemma 3.3 and its proof in [14], it is not difficult to see that relations (34)-(39) hold. The equivalence is also true, since

$$
\begin{aligned}
z & =x^{2}+y^{2}+(\tau-2)(x \circ y) \\
& =\left(x+\frac{\tau-2}{2} y\right)^{2}+\frac{\tau(4-\tau)}{4} y^{2} \\
& =\left(y+\frac{\tau-2}{2} x\right)^{2}+\frac{\tau(4-\tau)}{4} x^{2} \in \operatorname{bd} K^{n} .
\end{aligned}
$$

This completes the proof.

\section{The Jacobian Consistency}

In this section, we show the Jacobian consistency of the smoothing function $\phi_{\tau, \mathcal{\varepsilon}}$, which will play an important role for establishing the rapid convergence of smoothing methods. Moreover, we estimate the distance between the gradient of the smoothing functions and the subgradient of the oneparametric class of the SOC complementarity functions, which will help to adjust a parameter appropriately in smoothing methods.

It has been shown in Proposition 3.1 in [14] that the function $\phi_{\tau}$ with any $\tau \in(0,4)$ satisfies

$$
\phi_{\tau}(x, y)=0 \Longleftrightarrow x \in K^{n}, \quad y \in K^{n},\langle x, y\rangle=0 .
$$

Let $(x, y, \zeta) \in R^{n} \times R^{n} \times R^{l}$ and define

$$
\begin{gathered}
\Phi_{\tau, \mathcal{\varepsilon}}(x, y, \zeta):=\left(\begin{array}{c}
F(x, y, \zeta) \\
\phi_{\tau, \varepsilon}(x, y)
\end{array}\right), \\
\Phi_{\tau}(x, y, \zeta):=\left(\begin{array}{c}
F(x, y, \zeta) \\
\phi_{\tau}(x, y)
\end{array}\right) .
\end{gathered}
$$

It is easy to see that $\Phi_{\tau, \mathcal{\varepsilon}}(x, y, \zeta)=0$ is the perturbation of the system of equations $\Phi_{\tau}(x, y, \zeta)=0$. On account of (1), (42), and (43), we have

$$
\Phi_{\tau}(x, y, \zeta)=0 \Longleftrightarrow(x, y, \zeta) \text { solves }(1) .
$$

Since $\Phi_{\tau}(x, y, \zeta)$ is typically nonsmooth, we can solve approximately the smooth system $\Phi_{\tau, \varepsilon}(x, y, \zeta)=0$ by using Newton's method at each iteration, and then obtain a solution of $\Phi_{\tau}(x, y, \zeta)=0$ by reducing the parameter $\varepsilon$ to zero.

First, we show that the function $\Phi_{\tau, \varepsilon}(x, y, \zeta)$ satisfies the Jacobian consistency.

Lemma 5. For any $(x, y) \in R^{n} \times R^{n}$, one has

$$
\begin{aligned}
J_{\phi_{\tau}}^{0}(x, y) & :=\lim _{\varepsilon \rightarrow 0} \nabla \phi_{\tau, \varepsilon}(x, y) \\
& =\left(\begin{array}{c}
I-L\left(x+\frac{\tau-2}{2} y\right) J \\
I-L\left(y+\frac{\tau-2}{2} x\right) J
\end{array}\right),
\end{aligned}
$$

where

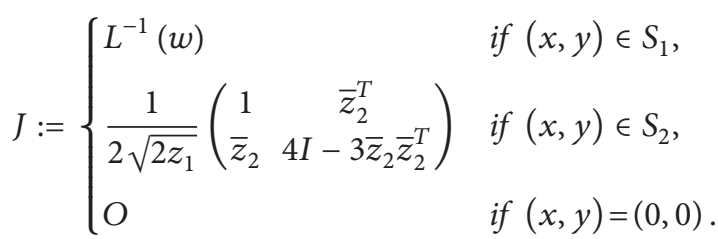

Proof. By (27) and the symmetry of $x$ and $y$, it suffices to show that

$$
\lim _{\varepsilon \rightarrow 0} L\left(x+\frac{\tau-2}{2} y\right) L^{-1}\left(w^{\varepsilon}\right)=L\left(x+\frac{\tau-2}{2} y\right) J .
$$

Case (i). If $(x, y) \in S_{1}$, we have from (18) that

$$
\lim _{\varepsilon \rightarrow 0} \lambda_{i}\left(z^{\varepsilon}\right)=\lim _{\varepsilon \rightarrow 0}\left[\lambda_{i}(z)+2 \varepsilon^{2}\right]=\lambda_{i}(z)>0,
$$

for $i=1,2$, and then,

$$
\begin{aligned}
\lim _{\varepsilon \rightarrow 0} w^{\varepsilon} & =\lim _{\varepsilon \rightarrow 0}\left[\sqrt{\lambda_{1}\left(z^{\varepsilon}\right)} u_{1}(z)+\sqrt{\lambda_{2}\left(z^{\varepsilon}\right)} u_{2}(z)\right] \\
& =\sqrt{\lambda_{1}(z)} u_{1}(z)+\sqrt{\lambda_{2}(z)} u_{2}(z) \\
& =w \in \operatorname{int} K^{n} .
\end{aligned}
$$

Therefore,

$$
\begin{array}{r}
\lim _{\varepsilon \rightarrow 0} L\left(x+\frac{\tau-2}{2} y\right) L^{-1}\left(w^{\varepsilon}\right) \\
\quad=L\left(x+\frac{\tau-2}{2} y\right) L^{-1}(w) .
\end{array}
$$


Case (ii). If $(x, y) \in S_{2}$, we obtain $(x, y) \neq(0,0)$, and (41) holds, and thus,

$$
\begin{gathered}
\left\|z_{2}\right\|=z_{1}=\left\|x+\frac{\tau-2}{2} y\right\|^{2}+\frac{\tau(4-\tau)}{4}\|y\|^{2}>0, \\
2 z_{1}=\lambda_{2}(z)>\lambda_{1}(z)=0 .
\end{gathered}
$$

Then we have from (18) that

$$
\begin{gathered}
\lambda_{1}\left(z^{\varepsilon}\right)=\lambda_{1}(z)+2 \varepsilon^{2}=2 \varepsilon^{2}>0, \\
\lambda_{2}\left(z^{\varepsilon}\right)=\lambda_{2}(z)+2 \varepsilon^{2}=2 z_{1}+2 \varepsilon^{2}>0 .
\end{gathered}
$$

For any $\varepsilon \neq 0$, it follows from (28) that $L^{-1}\left(w^{\varepsilon}\right)=L_{1}\left(z^{\varepsilon}\right)+$ $L_{2}\left(z^{\varepsilon}\right)$. We first show that $L(x+((\tau-2) / 2) y) L_{1}\left(z^{\varepsilon}\right)=O$ for any $\varepsilon \neq 0$. Let

$$
\omega:=\left(1 ; \bar{z}_{2}\right)=\frac{1}{\left\|z_{2}\right\|}\left(z_{1} ; z_{2}\right)=\frac{z}{z_{1}} .
$$

By (35), we have

$$
\left(x+\frac{\tau-2}{2} y\right) \circ \omega^{\prime}=\left(x+\frac{\tau-2}{2} y\right) \circ \frac{z^{\prime}}{z_{1}}=0,
$$

and therefore,

$$
\begin{aligned}
& L\left(x+\frac{\tau-2}{2} y\right) L_{1}\left(z^{\varepsilon}\right) \\
& =\frac{1}{2 \sqrt{\lambda_{1}\left(z^{\varepsilon}\right)}} L\left(x+\frac{\tau-2}{2} y\right) \omega^{\prime} \omega^{\prime T} \\
& =\frac{1}{2 \sqrt{2}|\varepsilon|}\left(x+\frac{\tau-2}{2} y\right) \circ \omega^{\prime} \omega^{\prime T} \\
& =O .
\end{aligned}
$$

We next show that $\lim _{\varepsilon \rightarrow 0} L_{2}\left(z^{\varepsilon}\right)=J$. In fact, we obtain from (52)

$$
\begin{aligned}
\lim _{\varepsilon \rightarrow 0} & L_{2}\left(z^{\varepsilon}\right) \\
= & \lim _{\varepsilon \rightarrow 0} \frac{1}{2 \sqrt{2 z_{1}+2 \varepsilon^{2}}}\left(\begin{array}{cc}
1 & \bar{z}_{2}^{T} \\
\bar{z}_{2} & \bar{z}_{2} \bar{z}_{2}^{T}
\end{array}\right) \\
& +\lim _{\varepsilon \rightarrow 0} \frac{2}{\sqrt{2 \varepsilon^{2}}+\sqrt{2 z_{1}+2 \varepsilon^{2}}}\left(\begin{array}{cc}
0 & 0^{T} \\
0 & I-\bar{z}_{2} \bar{z}_{2}^{T}
\end{array}\right), \\
= & \frac{1}{2 \sqrt{2 z_{1}}}\left(\begin{array}{cc}
1 & \bar{z}_{2}^{T} \\
\bar{z}_{2} & \bar{z}_{2} \bar{z}_{2}^{T}
\end{array}\right)+\frac{2}{\sqrt{2 z_{1}}}\left(\begin{array}{cc}
0 & 0^{T} \\
0 & I-\bar{z}_{2} \bar{z}_{2}^{T}
\end{array}\right), \\
= & \frac{1}{2 \sqrt{2 z_{1}}}\left(\begin{array}{cc}
1 & \bar{z}_{2}^{T} \\
\bar{z}_{2} & 4 I-3 \bar{z}_{2} \bar{z}_{2}^{T}
\end{array}\right)=J .
\end{aligned}
$$

Combining (55) and (56) yields

$$
\begin{aligned}
& \lim _{\varepsilon \rightarrow 0} L\left(x+\frac{\tau-2}{2} y\right) L^{-1}\left(w^{\varepsilon}\right) \\
& \quad=\lim _{\varepsilon \rightarrow 0} L\left(x+\frac{\tau-2}{2} y\right) L_{2}\left(z^{\varepsilon}\right) \\
& \quad=L\left(x+\frac{\tau-2}{2} y\right) J .
\end{aligned}
$$

Case (iii). If $(x, y)=(0,0)$, then $w^{\varepsilon}=\sqrt{z^{\varepsilon}}=\sqrt{2}|\varepsilon| e \in \operatorname{int} K^{n}$, and

$$
\begin{gathered}
\lim _{\varepsilon \rightarrow 0} L\left(x+\frac{\tau-2}{2} y\right) L^{-1}\left(w^{\varepsilon}\right) \\
=\lim _{\varepsilon \rightarrow 0} O \cdot \frac{1}{\sqrt{2}|\varepsilon|} e \\
=O=L\left(x+\frac{\tau-2}{2} y\right) J .
\end{gathered}
$$

This completes the proof.

Lemma 6. For any $(x, y) \in R^{n} \times R^{n}$, one has

$$
\left(\begin{array}{c}
I-U_{x} \\
I-U_{y}
\end{array}\right) \in \partial_{B} \phi_{\tau}(x, y),
$$

where

$$
\begin{aligned}
& U_{x}= \pm Z+L\left(x+\frac{\tau-2}{2} y\right) J, \\
& U_{y}= \pm \frac{\tau-2}{2} Z+L\left(y+\frac{\tau-2}{2} x\right) J,
\end{aligned}
$$

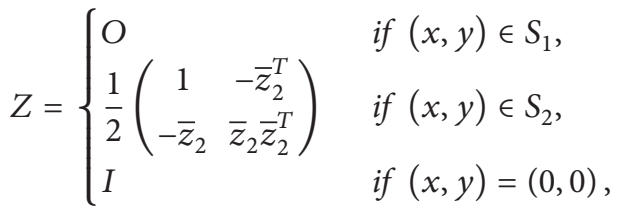

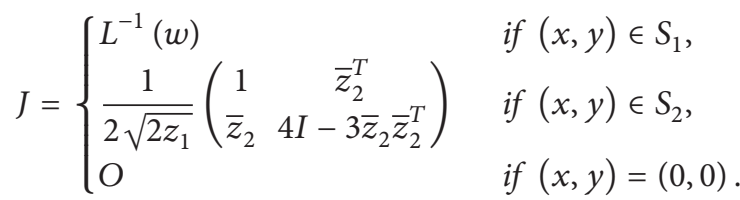

Proof. It follows from Proposition 5.2 [1] and the chain rule for differentiation that the one-parametric class of SOC complementarity functions $\phi_{\tau}$ is continuously differentiable at any $(x, y) \in S_{1}$ with

$\nabla \phi_{\tau}(x, y)$

$$
=\left(\begin{array}{c}
I-L\left(x+\frac{\tau-2}{2} y\right) L^{-1}(w) \\
I-L\left(y+\frac{\tau-2}{2} x\right) L^{-1}(w)
\end{array}\right) \in \partial_{B} \phi_{\tau}(x, y) .
$$

Therefore, it suffices to consider the two cases: $(x, y) \in S_{2}$ and $(x, y)=(0,0)$.

For any $(x, y) \in S_{2}$ or $(x, y)=(0,0)$, let $(\widehat{x}, y)=(x+\varepsilon e, y)$ with sufficiently small $\varepsilon \neq 0$, and define

$$
\begin{gathered}
\widehat{z}=\left(\widehat{z}_{1} ; \widehat{z}_{2}\right):=\widehat{x}^{2}+y^{2}+(\tau-2)(\widehat{x} \circ y), \\
\widehat{w}=\left(\widehat{w}_{1} ; \widehat{w}_{2}\right):=\sqrt{\widehat{z}}, \quad \widetilde{z}_{2}:=\frac{\widehat{z}_{2}}{\left\|\widehat{z}_{2}\right\|}, \\
\widehat{\lambda}_{i}=\lambda_{i}(\widehat{z}):=\widehat{z}_{1}+(-1)^{i}\left\|\widehat{z}_{2}\right\|, \quad i=1,2 .
\end{gathered}
$$


Direct calculations yield

$$
\begin{aligned}
\widehat{z}= & (x+\varepsilon e)^{2}+y^{2}+(\tau-2)(x+\varepsilon e) \circ y \\
= & x^{2}+y^{2}+(\tau-2)(x \circ y) \\
& +2 \varepsilon x+\varepsilon(\tau-2) y+\varepsilon^{2} e \\
= & z+2 \varepsilon x+\varepsilon(\tau-2) y+\varepsilon^{2} e .
\end{aligned}
$$

Therefore, we obtain

$$
\begin{gathered}
\widehat{z}_{1}=z_{1}+2 \varepsilon x_{1}+\varepsilon(\tau-2) y_{1}+\varepsilon^{2}, \\
\widehat{z}_{2}=z_{2}+2 \varepsilon x_{2}+\varepsilon(\tau-2) y_{2}, \\
\widehat{\lambda}_{i}=z_{1}+2 \varepsilon x_{1}+\varepsilon(\tau-2) y_{1}+\varepsilon^{2}+(-1)^{i} \\
\times\left\|z_{2}+2 \varepsilon x_{2}+\varepsilon(\tau-2) y_{2}\right\|, \quad i=1,2 .
\end{gathered}
$$

It is obvious that as $\varepsilon \rightarrow 0$, we have $(\widehat{x}, y) \rightarrow(x, y), \widehat{z} \rightarrow z$, $\widehat{w} \rightarrow w$, and $\hat{\lambda}_{i} \rightarrow \lambda_{i}(z)$ for $i=1,2$.

By the definition of $B$-subdifferential and (3), it suffices to show that

$$
\begin{aligned}
& \lim _{\varepsilon \rightarrow 0} L\left(\widehat{x}+\frac{\tau-2}{2} y\right) L^{-1}(\widehat{w})=U_{x}, \\
& \lim _{\varepsilon \rightarrow 0} L\left(y+\frac{\tau-2}{2} \widehat{x}\right) L^{-1}(\widehat{w})=U_{y},
\end{aligned}
$$

if $\phi_{\tau}$ is differentiable at $(\widehat{x}, y)$.

Case (i). If $(x, y)=(0,0)$, it is easy to see that $\widehat{z}=\varepsilon^{2} e \epsilon$ int $K^{n}, \widehat{w}=|\varepsilon| e$, and $\phi_{\tau}$ is differentiable at $(\widehat{x}, y)$. Then, we have

$$
\begin{aligned}
& \lim _{\varepsilon \rightarrow \pm 0} L\left(\widehat{x}+\frac{\tau-2}{2} y\right) L^{-1}(\widehat{w}) \\
& =\lim _{\varepsilon \rightarrow \pm 0} \varepsilon I \cdot \frac{1}{|\varepsilon|} I=\lim _{\varepsilon \rightarrow \pm 0} \operatorname{sgn}(\varepsilon) I \\
& = \pm I=U_{x}, \\
& \lim _{\varepsilon \rightarrow \pm 0} L\left(y+\frac{\tau-2}{2} \widehat{x}\right) L^{-1}(\widehat{w}) \\
& =\lim _{\varepsilon \rightarrow \pm 0} \frac{\tau-2}{2} \varepsilon I \cdot \frac{1}{|\varepsilon|} I=\lim _{\varepsilon \rightarrow \pm 0} \frac{\tau-2}{2} \operatorname{sgn}(\varepsilon) I \\
& = \pm \frac{\tau-2}{2} I=U_{y} .
\end{aligned}
$$

Case (ii). If $(x, y) \in S_{2}$, we obtain $z \in$ bd $K^{n} \backslash\{0\}$, and thus, from (38) and (39),

$$
\begin{aligned}
\left\|\widehat{z}_{2}\right\|^{2}= & \left\|z_{2}+2 \varepsilon x_{2}+\varepsilon(\tau-2) y_{2}\right\|^{2} \\
= & \left\|z_{2}\right\|^{2}+4 \varepsilon^{2}\left\|x_{2}\right\|^{2}+\varepsilon^{2}(\tau-2)^{2}\left\|y_{2}\right\|^{2}+4 \varepsilon z_{2}^{T} x_{2} \\
& +2 \varepsilon(\tau-2) z_{2}^{T} y_{2}+4 \varepsilon^{2}(\tau-2) x_{2}^{T} y_{2} \\
= & z_{1}^{2}+4 \varepsilon^{2} x_{1}^{2}+\varepsilon^{2}(\tau-2)^{2} y_{1}^{2}+4 \varepsilon z_{1} x_{1} \\
& +2 \varepsilon(\tau-2) z_{1} y_{1}+4 \varepsilon^{2}(\tau-2) x_{1} y_{1} \\
= & {\left[z_{1}+2 \varepsilon x_{1}+\varepsilon(\tau-2) y_{1}\right]^{2} }
\end{aligned}
$$

Since $z_{1}>0$ and $\varepsilon$ is sufficiently small, we obtain

$$
\left\|\widehat{z}_{2}\right\|=z_{1}+2 \varepsilon x_{1}+\varepsilon(\tau-2) y_{1}>0 .
$$

By (66) and (70), we have

$$
\begin{aligned}
\hat{\lambda}_{1} & =z_{1}+2 \varepsilon x_{1}+\varepsilon(\tau-2) y_{1}+\varepsilon^{2}-\left\|\widehat{z}_{2}\right\| \\
& =\varepsilon^{2}>0, \\
\hat{\lambda}_{2} & =z_{1}+2 \varepsilon x_{1}+\varepsilon(\tau-2) y_{1}+\varepsilon^{2}+\left\|\widehat{z}_{2}\right\| \\
& =2\left[z_{1}+2 \varepsilon x_{1}+\varepsilon(\tau-2) y_{1}\right]+\varepsilon^{2} \\
& >0 .
\end{aligned}
$$

Relations (71) and (72) imply that $\widehat{z} \in$ int $K^{n}$, and thus, $\phi_{\tau}$ is differentiable at $(\widehat{x}, y)$.

Now we will show that

$$
\lim _{\varepsilon \rightarrow 0} L\left(\widehat{x}+\frac{\tau-2}{2} y\right) L^{-1}(\widehat{w})=U_{x}
$$

By Proposition 5.2 in [1], we have $L^{-1}(\widehat{w})=L_{1}(\widehat{z})+L_{2}(\widehat{z})$, where $L_{1}(\widehat{z})$ and $L_{2}(\widehat{z})$ are given by (29) with $\widehat{z}$ and $\widetilde{z}_{2}$ replacing $z^{\varepsilon}$ and $\bar{z}_{2}$, respectively. By $(70)$,

$$
\begin{gathered}
\widehat{\omega}:=\left(1 ; \widetilde{z}_{2}\right)=\frac{1}{\left\|\widehat{z}_{2}\right\|}\left(\left\|\widehat{z}_{2}\right\| ; \widehat{z}_{2}\right) \\
=\frac{1}{\left\|\widehat{z}_{2}\right\|}\left(\left\|z_{2}+2 \varepsilon x_{2}+\varepsilon(\tau-2) y_{2}\right\| ;\right. \\
\left.z_{2}+2 \varepsilon x_{2}+\varepsilon(\tau-2) y_{2}\right) \\
=\frac{1}{\left\|\widehat{z}_{2}\right\|}\left(z_{1}+2 \varepsilon x_{1}+\varepsilon(\tau-2) y_{1} ;\right. \\
\left.z_{2}+2 \varepsilon x_{2}+\varepsilon(\tau-2) y_{2}\right) \\
=\frac{1}{\left\|\widehat{z}_{2}\right\|}[z+2 \varepsilon x+\varepsilon(\tau-2) y] .
\end{gathered}
$$


According to (35), (39), (71), and (74), we have

$$
\begin{aligned}
(x+ & \left.\frac{\tau-2}{2} y\right) \circ \widehat{\omega}^{\prime} \\
= & \frac{1}{\left\|\widehat{z}_{2}\right\|}\left(x+\frac{\tau-2}{2} y\right) \\
& \circ[z+2 \varepsilon x+\varepsilon(\tau-2) y]^{\prime} \\
= & \frac{1}{\left\|\widehat{z}_{2}\right\|}\left[\left(x+\frac{\tau-2}{2} y\right) \circ z^{\prime}\right. \\
& \left.\quad+2 \varepsilon\left(x+\frac{\tau-2}{2} y\right) \circ\left(x+\frac{\tau-2}{2} y\right)^{\prime}\right]
\end{aligned}
$$

$$
=0 \text {, }
$$

and thus,

$$
\begin{aligned}
& L\left(\widehat{x}+\frac{\tau-2}{2} y\right) L_{1}(\widehat{z}) \\
& \quad=\frac{1}{2 \sqrt{\widehat{\lambda}_{1}}}\left(x+\varepsilon e+\frac{\tau-2}{2} y\right) \circ \widehat{\omega}^{\prime} \widehat{\omega}^{\prime T} \\
& \quad=\frac{1}{2|\varepsilon|}\left[\left(x+\frac{\tau-2}{2} y\right) \circ \widehat{\omega}^{\prime} \widehat{\omega}^{\prime T}+\varepsilon \widehat{\omega}^{\prime} \widehat{\omega}^{\prime T}\right] \\
& =\frac{\operatorname{sgn}(\varepsilon)}{2} \widehat{\omega}^{\prime} \widehat{\omega}^{\prime T} \\
& =\frac{\operatorname{sgn}(\varepsilon)}{2}\left(\begin{array}{cc}
1 & -\widetilde{z}_{2}^{T} \\
-\widetilde{z}_{2} & \left.\widetilde{z}_{2} \widetilde{z}_{2}^{T}\right) .
\end{array}\right.
\end{aligned}
$$

It follows from (70), (71), (72), and (74) that $\hat{\lambda}_{1} \rightarrow \lambda_{1}(z)=$ $0, \hat{\lambda}_{2} \rightarrow \lambda_{2}(z)=2 z_{1}$, and $\widetilde{z}_{2} \rightarrow \bar{z}_{2}$ as $\varepsilon \rightarrow 0$. Then, by following the proof of Case (ii) in Lemma 5 , we obtain

$$
\lim _{\varepsilon \rightarrow 0} L_{2}(\widehat{z})=\frac{1}{2 \sqrt{2 z_{1}}}\left(\begin{array}{cc}
1 & \bar{z}_{2}^{T} \\
\bar{z}_{2} & 4 I-3 \bar{z}_{2} \bar{z}_{2}^{T}
\end{array}\right)=J .
$$

Hence, we have from (76) and (77) that

$$
\begin{aligned}
\lim _{\varepsilon \rightarrow \pm 0} L\left(\widehat{x}+\frac{\tau-2}{2} y\right) L^{-1}(\widehat{w}) \\
=\lim _{\varepsilon \rightarrow \pm 0} L\left(\widehat{x}+\frac{\tau-2}{2} y\right) L_{1}(\widehat{z}) \\
\quad+\lim _{\varepsilon \rightarrow \pm 0} L\left(\widehat{x}+\frac{\tau-2}{2} y\right) L_{2}(\widehat{z}) \\
=\lim _{\varepsilon \rightarrow \pm 0} \frac{\operatorname{sgn}(\varepsilon)}{2} \widehat{\omega}^{\prime} \widehat{\omega}^{\prime T} \\
\quad+\lim _{\varepsilon \rightarrow \pm 0} L\left(\widehat{x}+\frac{\tau-2}{2} y\right) J \\
= \pm \\
+Z+L\left(x+\frac{\tau-2}{2} y\right) J=U_{x} .
\end{aligned}
$$

Next, we will show that

$$
\lim _{\varepsilon \rightarrow 0} L\left(y+\frac{\tau-2}{2} \widehat{x}\right) L^{-1}(\widehat{w})=U_{y} .
$$

By (38), (39), and (74), we have

$$
\begin{aligned}
(y+ & \left.\frac{\tau-2}{2} x\right) \circ \widehat{\omega}^{\prime} \\
= & \frac{1}{\left\|\widehat{z}_{2}\right\|}\left(y+\frac{\tau-2}{2} x\right) \\
& \circ[z+2 \varepsilon x+\varepsilon(\tau-2) y]^{\prime} \\
= & \frac{1}{\left\|\widehat{z}_{2}\right\|}\left[\left(y+\frac{\tau-2}{2} x\right) \circ z^{\prime}+2 \varepsilon\left(y+\frac{\tau-2}{2} x\right)\right. \\
= & 0,
\end{aligned}
$$

and then,

$$
\begin{aligned}
& L\left(y+\frac{\tau-2}{2} \widehat{x}\right) L_{1}(\widehat{z}) \\
& \quad=\frac{1}{2 \sqrt{\widehat{\lambda}_{1}}}\left(y+\frac{\tau-2}{2} x+\frac{\tau-2}{2} \varepsilon e\right) \circ \widehat{\omega}^{\prime} \widehat{\omega}^{\prime T} \\
& =\frac{1}{2|\varepsilon|}\left[\left(y+\frac{\tau-2}{2} x\right) \circ \widehat{\omega}^{\prime} \widehat{\omega}^{\prime T}+\frac{\tau-2}{2} \varepsilon \widehat{\omega}^{\prime} \widehat{\omega}^{\prime T}\right] \\
& =\frac{\tau-2}{4} \operatorname{sgn}(\varepsilon) \widehat{\omega}^{\prime} \widehat{\omega}^{\prime T} \\
& =\frac{\tau-2}{4} \operatorname{sgn}(\varepsilon)\left(\begin{array}{cc}
1 & -\widetilde{z}_{2}^{T} \\
-\widetilde{z}_{2} & \widetilde{z}_{2} \widetilde{z}_{2}^{T}
\end{array}\right) .
\end{aligned}
$$

Hence, we have from (77) and (81) that

$$
\begin{aligned}
\lim _{\varepsilon \rightarrow \pm 0} L & \left(y+\frac{\tau-2}{2} \widehat{x}\right) L^{-1}(\widehat{w}) \\
= & \lim _{\varepsilon \rightarrow \pm 0} L\left(y+\frac{\tau-2}{2} \widehat{x}\right) L_{1}(\widehat{z}) \\
& +\lim _{\varepsilon \rightarrow \pm 0} L\left(y+\frac{\tau-2}{2} \widehat{x}\right) L_{2}(\widehat{z}) \\
= & \lim _{\varepsilon \rightarrow \pm 0} \frac{\tau-2}{4} \operatorname{sgn}(\varepsilon) \widehat{\omega}^{\prime} \widehat{\omega}^{\prime T} \\
& +\lim _{\varepsilon \rightarrow \pm 0} L\left(y+\frac{\tau-2}{2} \widehat{x}\right) J \\
= & \pm \frac{\tau-2}{2} Z+L\left(y+\frac{\tau-2}{2} x\right) J=U_{y} .
\end{aligned}
$$

This completes the proof.

Theorem 7. The function $\Phi_{\tau, \varepsilon}$ defined by (43) with $\varepsilon>0$ satisfies the Jacobian consistency.

Proof. By (43), it suffices to show the Jacobian consistency of the function $\phi_{\tau, \varepsilon}$ with $\varepsilon>0$. Let

$$
V^{i}:=\left(\begin{array}{c}
I-U_{x}^{i} \\
I-U_{y}^{i}
\end{array}\right),
$$

where

$$
\begin{gathered}
U_{x}^{i}=(-1)^{i} Z+L\left(x+\frac{\tau-2}{2} y\right) J, \\
U_{y}^{i}=(-1)^{i} \frac{\tau-2}{2} Z+L\left(y+\frac{\tau-2}{2} x\right) J,
\end{gathered}
$$


for $i=1,2$, and $Z$ and $J$ are given by (61). Define

$$
\begin{aligned}
V & :=\frac{1}{2}\left(V^{1}+V^{2}\right) \\
& =\left(\begin{array}{c}
I-L\left(x+\frac{\tau-2}{2} y\right) J \\
I-L\left(y+\frac{\tau-2}{2} x\right) J
\end{array}\right) .
\end{aligned}
$$

On the one hand, we obtain from Lemma 5 that $V=$ $J_{\phi_{\tau}}^{0}(x, y)=\lim _{\varepsilon \rightarrow 0} \nabla \phi_{\tau, \varepsilon}(x, y)$. On the other hand, we have by Lemma 6 that $V^{1}, V^{2} \in \partial_{B} \phi_{\tau}(x, y)$, and therefore, $V=$ $(1 / 2)\left(V^{1}+V^{2}\right) \in \partial \phi_{\tau}(x, y)$. This together with Theorem 3 and Definition 1 implies the Jacobian consistency of $\phi_{\tau, \varepsilon}$ with $\varepsilon>0$.

Now, we are in a position to estimate an upper bound of the parameter $\varepsilon>0$ for the predicted accuracy of the distance between the gradient of $\Phi_{\tau, \varepsilon}$ and the subgradient of $\Phi_{\tau}$.

Theorem 8. Let $\tau \in(0,4), \delta>0$ be given, and $(x, y, \zeta) \in$ $R^{2 n+l}$ be any point. Let $\theta_{\tau}(x, y)$ be any function such that

$$
\theta_{\tau}(x, y) \geq \| \begin{aligned}
& L\left(x+\frac{\tau-2}{2} y\right) J \| \\
& L\left(y+\frac{\tau-2}{2} x\right) J \|
\end{aligned}
$$

and let $\bar{\varepsilon}: R^{2 n} \times R_{+} \rightarrow R_{+} \cup\{+\infty\}$ be defined by

$$
\bar{\varepsilon}(x, y, \delta):=\left\{\begin{array}{l}
\frac{\lambda_{1}(z) \delta}{\sqrt{2\left(\theta_{\tau}(x, y)^{2}-\lambda_{1}(z) \delta^{2}\right)}} \\
\quad \text { if }(x, y) \in S_{1}, \delta<\frac{\theta_{\tau}(x, y)}{\sqrt{\lambda_{1}(z)}}, \\
\frac{z_{1} \delta}{2 \sqrt{\theta_{\tau}(x, y)\left(2 \theta_{\tau}(x, y)-\delta \sqrt{2 z_{1}}\right)}} \\
\quad \text { if }(x, y) \in S_{2}, \delta<\frac{2 \theta_{\tau}(x, y)}{\sqrt{2 z_{1}}}, \\
+\infty \quad \text { otherwise. }
\end{array}\right.
$$

Then, for any $\varepsilon \in R$ such that $0<|\varepsilon| \leq \bar{\varepsilon}(x, y, \delta)$, we have

$$
\operatorname{dist}\left(\nabla \Phi_{\tau, \varepsilon}(x, y, \zeta), \partial \Phi_{\tau}(x, y, \zeta)\right)<\delta
$$

Proof. Since it follows from the proof of Theorem 7 that $J_{\phi_{\tau}}^{0}(x, y)=V \in \partial \phi_{\tau}(x, y)$ for any $(x, y, \zeta) \in R^{2 n+l}$, we obtain

$$
\begin{aligned}
& J_{\Phi_{\tau}}^{0}(x, y, \zeta) \\
& \quad:=\left(\begin{array}{cc}
J_{\phi_{\tau}}^{0}(x, y) & \nabla_{x, y} F(x, y, \zeta) \\
O & \nabla_{\zeta} F(x, y, \zeta)
\end{array}\right) \in \partial \Phi_{\tau}(x, y, \zeta) .
\end{aligned}
$$

Therefore, we have from (27) and (85) that

$$
\begin{aligned}
\operatorname{dist} & \left(\nabla \Phi_{\tau, \varepsilon}(x, y, \zeta), \partial \Phi_{\tau}(x, y, \zeta)\right) \\
= & \min \left\{\left\|\nabla \Phi_{\tau, \varepsilon}(x, y, \zeta)-W\right\|: W \in \partial \Phi_{\tau}(x, y, \zeta)\right\} \\
\leq & \left\|\nabla \Phi_{\tau, \varepsilon}(x, y, \zeta)-J_{\Phi_{\tau}}^{0}(x, y, \zeta)\right\|
\end{aligned}
$$

$$
\begin{aligned}
& =\left\|\nabla \phi_{\tau, \varepsilon}(x, y, \zeta)-J_{\phi_{\tau}}^{0}(x, y, \zeta)\right\| \\
& =\left\|\begin{array}{l}
\left(x+\frac{\tau-2}{2} y\right)\left(L^{-1}(\widehat{w})-J\right) \| \\
L\left(y+\frac{\tau-2}{2} x\right)\left(L^{-1}(\widehat{w})-J\right)
\end{array}\right\| .
\end{aligned}
$$

Then, by following the proof of Proposition 4.1 in [12], we obtain

$$
\begin{aligned}
& \operatorname{dist}\left(\nabla \Phi_{\tau, \varepsilon}(x, y, \zeta), \partial \Phi_{\tau}(x, y, \zeta)\right) \\
& \leq {\left[h_{\tau, 0}(x, y)-h_{\tau, \varepsilon}(x, y)\right] } \\
& \times\left\|L\left(x+\frac{\tau-2}{2} y\right) J\right\| \\
& L\left(y+\frac{\tau-2}{2} x\right) J \|
\end{aligned}
$$

where $h_{\tau, \varepsilon}: R^{2 n} \rightarrow R_{+}$is defined as

$$
h_{\tau, \varepsilon}(x, y):= \begin{cases}\frac{1}{\sqrt{\lambda_{1}(z)+2 \varepsilon^{2}}} & \text { if }(x, y) \in S_{1}, \\ \frac{\sqrt{2}}{\sqrt{z_{1}+\varepsilon^{2}}+|\varepsilon|} & \text { if }(x, y) \in S_{2}, \\ 0 & \text { if }(x, y)=(0,0) .\end{cases}
$$

Hence, similar to the proof of Theorem 4.1 in [12], we have the desired result.

\section{An Alternative Proof}

In this section, we study the directional derivative and $B$ subdifferential of the one-parametric class of SOC complementarity functions $\phi_{\tau}$. Based on these results, we present an alternative way to prove the Jacobian consistency of the oneparametric class of smoothing functions $\phi_{\tau, \varepsilon}$.

By Corollary 3.3 in [18], it is not difficult to see that the function $\phi_{\tau}$ given as (3) is directionally differentiable everywhere. However, as far as we know, the expression of its directional derivative is not given in the available literature. In this section, we derive its expression and prove that the $B$ subdifferential of $\phi_{\tau}(x, y)$ at a general point coincides with that of its directional derivative function at the origin.

In light of the $B$-subdifferential of $\phi_{\mathrm{FB}}(x, y)[4,10,19]$, we obtain the following four results, which can be shown by following the proofs of Proposition 9, Lemma 11, Lemma 12, and Proposition 13 in [4], respectively.

Proposition 9. For any given $x=\left(x_{1} ; x_{2}\right), y=\left(y_{1} ; y_{2}\right) \in$ $R \times R^{n-1}$, the directional derivative $\phi_{\tau}^{\prime}((x, y) ;(g, h))$ of $\phi_{\tau}$ at $(x, y)$ has the following form.

(i) If $(x, y)=(0,0)$, then $\phi_{\tau}^{\prime}((x, y) ;(g, h))=\phi_{\tau}(g, h)$.

(ii) If $(x, y) \in S_{1}$, then

$$
\begin{aligned}
\phi_{\tau}^{\prime}((x, y) ;(g, h))= & {\left[I-L^{-1}(w) L\left(x+\frac{\tau-2}{2} y\right)\right] g } \\
& +\left[I-L^{-1}(w) L\left(y+\frac{\tau-2}{2} x\right)\right] h .
\end{aligned}
$$




$$
\begin{aligned}
& \phi_{\tau}^{\prime}((x, y) ;(g, h))=g+h-\frac{\varphi(g, h)}{2}\left(\begin{array}{c}
1 \\
-\bar{z}_{2}
\end{array}\right) \\
& +\frac{\left(x_{2}+((\tau-2) / 2) y_{2}\right)^{T} g_{2}+\left(y_{2}+((\tau-2) / 2) x_{2}\right)^{T} h_{2}}{2 \sqrt{x_{1}^{2}+y_{1}^{2}+(\tau-2) x_{1} y_{1}}}\left(\frac{0}{z_{2}}\right)-\frac{1}{2 \sqrt{x_{1}^{2}+y_{1}^{2}+(\tau-2) x_{1} y_{1}}} \\
& \times\left(\begin{array}{c}
\left(x+\frac{\tau-2}{2} y\right)^{T} g+\left(y+\frac{\tau-2}{2} x\right)^{T} h \\
2\left(x_{1}+\frac{\tau-2}{2} y_{1}\right) g_{2}+g_{1}\left(x_{2}+\frac{\tau-2}{2} y_{2}\right)+2\left(y_{1}+\frac{\tau-2}{2} x_{1}\right) h_{2}+h_{1}\left(y_{2}+\frac{\tau-2}{2} x_{2}\right)
\end{array}\right),
\end{aligned}
$$

where $g=\left(g_{1} ; g_{2}\right), h=\left(h_{1} ; h_{2}\right) \in R \times R^{n-1}, \bar{z}_{2}$ is given as (20), and the function $\varphi: R^{n} \times R^{n} \rightarrow R$ is defined by

$$
\varphi(g, h)=\frac{\sqrt{\left(\theta_{1}(g, h)\right)^{2}+\left\|\theta_{2}(g, h)\right\|^{2}}}{\sqrt{x_{1}^{2}+y_{1}^{2}+(\tau-2) x_{1} y_{1}}},
$$

$$
\begin{aligned}
\theta_{1}(g, h)= & \left(x_{1}+\frac{\tau-2}{2} y_{1}\right) g_{1}-\left(x_{2}+\frac{\tau-2}{2} y_{2}\right)^{T} g_{2} \\
& +\left(y_{1}+\frac{\tau-2}{2} x_{1}\right) h_{1}-\left(y_{2}+\frac{\tau-2}{2} x_{2}\right)^{T} h_{2} \\
\theta_{2}(g, h)= & \left(x_{1}+\frac{\tau-2}{2} y_{1}\right) h_{2}-h_{1}\left(x_{2}+\frac{\tau-2}{2} y_{2}\right) \\
& +g_{1}\left(y_{2}+\frac{\tau-2}{2} x_{2}\right)-\left(y_{1}+\frac{\tau-2}{2} x_{1}\right) g_{2} .
\end{aligned}
$$

Lemma 10. For any given $(x, y) \in S_{2}$, let $\varphi$ be defined by (95).

with

$\partial_{B} \varphi(0,0)$

$$
=\left\{\begin{array}{l}
{\left[\begin{array}{l}
\left.\left(\frac{\zeta_{2}^{T}\left(y_{2}+((\tau-2) / 2) x_{2}\right)+\zeta_{1}\left(x_{1}+((\tau-2) / 2) y_{1}\right)}{\sqrt{x_{1}^{2}+y_{1}^{2}+(\tau-2) x_{1} y_{1}}} ; \frac{-\left(y_{1}+((\tau-2) / 2) x_{1}\right) \zeta_{2}-\zeta_{1}\left(x_{2}+((\tau-2) / 2) y_{2}\right)}{\sqrt{x_{1}^{2}+y_{1}^{2}+(\tau-2) x_{1} y_{1}}}\right)\right] \\
\left(\frac{-\zeta_{2}^{T}\left(x_{2}+((\tau-2) / 2) y_{2}\right)+\zeta_{1}\left(y_{1}+((\tau-2) / 2) x_{1}\right)}{\sqrt{x_{1}^{2}+y_{1}^{2}+(\tau-2) x_{1} y_{1}}} ; \frac{\left(x_{1}+((\tau-2) / 2) y_{1}\right) \zeta_{2}-\zeta_{1}\left(y_{2}+((\tau-2) / 2) x_{2}\right)}{\sqrt{x_{1}^{2}+y_{1}^{2}+(\tau-2) x_{1} y_{1}}}\right)
\end{array}\right]}
\end{array}\right.
$$

$$
\left.\zeta=\left(\zeta_{1} ; \zeta_{2}\right) \in R \times R^{n-1} \text { satisfying } \zeta_{1}^{2}+\left\|\zeta_{2}\right\|^{2}=1\right\}
$$

Lemma 11. For any given $(x, y) \in R^{n} \times R^{n}$, let $\psi_{\tau}(\cdot, \cdot) \equiv$ $\phi_{\tau}^{\prime}((x, y) ;(\cdot, \cdot))$. Then,

$$
\partial_{B} \phi_{\tau}(x, y)=\partial_{B} \psi_{\tau}(0,0)
$$

Proposition 12. For any given $(x, y) \in S_{2}$, we have $\partial_{B} \phi_{\tau}(x, y)$ $=\left\{\left(\begin{array}{l}I-X-p u_{1}(z)^{T} \\ I-Y-q u_{1}(z)^{T}\end{array}\right) \mid p=\left(p_{1} ; p_{2}\right)\right.$, 


$$
\begin{aligned}
& q=\left(q_{1} ; q_{2}\right) \text { satisfying } \\
& p=\left(\frac{\zeta_{2}^{T}\left(y_{2}+((\tau-2) / 2) x_{2}\right)+\zeta_{1}\left(x_{1}+((\tau-2) / 2) y_{1}\right)}{\sqrt{x_{1}^{2}+y_{1}^{2}+(\tau-2) x_{1} y_{1}}} ;\right. \\
&\left.\frac{-\left(y_{1}+((\tau-2) / 2) x_{1}\right) \zeta_{2}-\zeta_{1}\left(x_{2}+((\tau-2) / 2) y_{2}\right)}{\sqrt{x_{1}^{2}+y_{1}^{2}+(\tau-2) x_{1} y_{1}}}\right),
\end{aligned}
$$

$$
\left.\begin{array}{c}
q=\left(\frac{-\zeta_{2}^{T}\left(x_{2}+((\tau-2) / 2) y_{2}\right)+\zeta_{1}\left(y_{1}+((\tau-2) / 2) x_{1}\right)}{\sqrt{x_{1}^{2}+y_{1}^{2}+(\tau-2) x_{1} y_{1}}} ;\right. \\
\left.\quad \frac{\left(x_{1}+\left(\frac{\tau-2}{2}\right) y_{1}\right) \zeta_{2}-\zeta_{1}\left(y_{2}+\left(\frac{\tau-2}{2}\right) x_{2}\right)}{\sqrt{x_{1}^{2}+y_{1}^{2}+(\tau-2) x_{1} y_{1}}}\right), \\
\text { for some } \zeta=\left(\zeta_{1} ; \zeta_{2}\right) \text { such that } \zeta_{1}^{2}+\left\|\zeta_{2}\right\|^{2}=1
\end{array}\right\},
$$

where $u_{1}(z)$ and $\bar{z}_{2}$ are defined by (19) and (20), respectively, and

$$
\begin{aligned}
X= & \frac{1}{2 \sqrt{x_{1}^{2}+y_{1}^{2}+(\tau-2) x_{1} y_{1}}} \\
& \times\left(\begin{array}{l}
x_{1}+\frac{\tau-2}{2} y_{1} \\
x_{2}^{T}+\frac{\tau-2}{2} y_{2} 2\left(x_{1}+\frac{\tau-2}{2} y_{1}\right) I-\left(x_{2}+\frac{\tau-2}{2} y_{2}\right) \bar{z}_{2}^{T}
\end{array}\right), \\
Y= & \frac{2 \sqrt{x_{1}^{2}+y_{1}^{2}+(\tau-2) x_{1} y_{1}}}{x_{1}} \quad y_{2}^{T}+\frac{\tau-2}{2} x_{2}^{T} \\
& \times\left(\begin{array}{l}
y_{1}+\frac{\tau-2}{2} x_{1} \\
y_{2}+\frac{\tau-2}{2} x_{2}
\end{array} 2\left(y_{1}+\frac{\tau-2}{2} x_{1}\right) I-\left(y_{2}+\frac{\tau-2}{2} x_{2}\right) \bar{z}_{2}^{T}\right)
\end{aligned}
$$

Theorem 13. The function $\phi_{\tau, \varepsilon}$ defined by (13) with $\varepsilon>0$ satisfies the Jacobian consistency.

Proof. By the proof of Theorem 7, it suffices to show that $V \in$ $\partial \phi_{\tau}(x, y)$, where $V$ is defined by (85). From Proposition 9 and Lemma 11, it is obvious that the result holds if $(x, y) \in S_{1}$ or $(x, y)=(0,0)$. Now, we prove $V \in \partial \phi_{\tau}(x, y)$ if $(x, y) \in S_{2}$. From Proposition 12, it is not difficult to see that

$$
\left(\begin{array}{l}
I-X \\
I-Y
\end{array}\right) \in \partial \phi_{\tau}(x, y)
$$

for any given $(x, y) \in S_{2}$. Direct calculations together with Lemma 4 yield

$$
\begin{aligned}
& X=L\left(x+\frac{\tau-2}{2} y\right) J, \\
& Y=L\left(y+\frac{\tau-2}{2} x\right) J,
\end{aligned}
$$

if $(x, y) \in S_{2}$. Therefore, it follows from (101) that for any given $(x, y) \in S_{2}$,

$$
V \equiv\left(\begin{array}{l}
I-X \\
I-Y
\end{array}\right) \in \partial \phi_{\tau}(x, y),
$$

which completes the proof.

\section{Conclusions}

In this paper, we show the Jacobian consistency of the oneparametric class of smoothing functions $\phi_{\tau, \varepsilon}$, which will play an important role for establishing the rapid convergence of smoothing methods. Moreover, we estimate the distance between the gradient of the smoothing functions $\phi_{\tau, \varepsilon}$ and the subgradient of the one-parametric class of the SOC complementarity functions $\phi_{\tau}$, which will help to adjust a parameter appropriately in smoothing methods. The related topics for further study are about smoothing methods for solving the SOCCP via the Jacobian consistency of the oneparametric class of smoothing functions.

\section{Acknowledgments}

This research is supported by the National Natural Science Foundation of China (no. 71171150), China Postdoctoral Science Foundation (no. 2012M511651), and the Excellent Youth Project of Hubei Provincial Department of Education (no. Q20122709), China. The authors are grateful to the editor and the anonymous referees for their valuable comments on this paper. In particular, the authors thank one of the referees 
and Professor Shaohua Pan for their helpful suggestions on the alternative proof of the Jacobian consistency, which have greatly improved this paper.

\section{References}

[1] M. Fukushima, Z. Q. Luo, and P. Tseng, "Smoothing functions for second-order-cone complementarity problems," SIAM Journal on Optimization, vol. 12, no. 2, pp. 436-460, 2002.

[2] J. Zhou, J. S. Chen, and G. M. Lee, "On set-valued complementarity problems," Abstract and Applied Analysis, vol. 2013, Article ID 105930, 11 pages, 2013.

[3] F. Alizadeh and D. Goldfarb, "Second-order cone programming," Mathematical Programming B, vol. 95, no. 1, pp. 3-51, 2003.

[4] S. Pan, S. Bi, and J. S. Chen, "Nonsingular conditions for FB system of reformulating nonlinear second-order cone programming," Abstract and Applied Analysis, vol. 2013, Article ID 602735, 21 pages, 2013.

[5] M. S. Lobo, L. Vandenberghe, S. Boyd, and H. Lebret, "Applications of second-order cone programming," Linear Algebra and Its Applications, vol. 284, no. 1-3, pp. 193-228, 1998.

[6] Y. J. Kuo and H. D. Mittelmann, "Interior point methods for second-order cone programming and OR applications," Computational Optimization and Applications, vol. 28, no. 3, pp. 255-285, 2004.

[7] S. Hayashi, N. Yamashita, and M. Fukushima, "A combined smoothing and regularization method for monotone secondorder cone complementarity problems," SIAM Journal on Optimization, vol. 15, no. 2, pp. 593-615, 2005.

[8] J. S. Chen and P. Tseng, "An unconstrained smooth minimization reformulation of the second-order cone complementarity problem," Mathematical Programming, vol. 104, no. 2-3, pp. 293327, 2005.

[9] L. Qi, D. Sun, and G. Zhou, "A new look at smoothing Newton methods for Nonlinear Complementarity Problems and box constrained variational inequalities," Mathematical Programming B, vol. 87, no. 1, pp. 1-35, 2000.

[10] J. S. Chen and S. Pan, "A survey on SOC complementarity functions and solution methods for SOCPs and SOCCPs," Pacific Journal of Optimization, vol. 8, pp. 33-74, 2012.

[11] J. S. Chen, X. Chen, and P. Tseng, "Analysis of nonsmooth vector-valued functions associated with second-order cones," Mathematical Programming, vol. 101, no. 1, pp. 95-117, 2004.

[12] H. Ogasawara and Y. Narushima, "The Jacobian consistency of a smoothed Fischer-Burmeister function associated with secondorder cones," Journal of Mathematical Analysis and Applications, vol. 394, pp. 231-247, 2012.

[13] J. S. Chen, S. Pan, and T. C. Lin, "A smoothing Newton method based on the generalized Fischer-Burmeister function for MCPs," Nonlinear Analysis, Theory, Methods and Applications, vol. 72, no. 9-10, pp. 3739-3758, 2010.

[14] J. S. Chen and S. Pan, "A one-parametric class of merit functions for the second-order cone complementarity problem," Computational Optimization and Applications, vol. 45, no. 3, pp. 581606, 2010.

[15] U. Faraut and A. Korányi, Analysis on Symmetric Cones, Oxford University Press, Oxford Mathematical Monographs, New Yor, NY, USA, 1994.

[16] X. Chen, L. Qi, and D. Sun, "Global and superlinear convergence of the smoothing newton method and its application to general box constrained variational inequalities," Mathematics of Computation, vol. 67, no. 222, pp. 519-540, 1998.

[17] F. H. Clarke, Optimization and Nonsmooth Analysis, John Wiley and Sons, New York, NY, USA, 1983.

[18] D. Sun and J. Sun, "Strong semismoothness of the FischerBurmeister SDC and SOC complementarity functions," Mathematical Programming, vol. 103, no. 3, pp. 575-581, 2005.

[19] L. Han, S. Bi, and S. Pan, "Nonsingularity of FB system and constraint nondegeneracy in semidefinite programming," Numerical Algorithms, vol. 62, no. 1, pp. 79-113, 2012. 


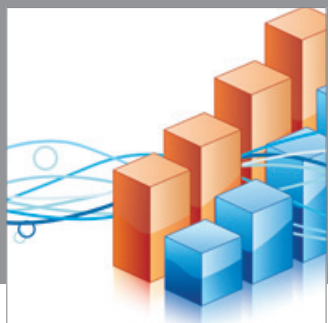

Advances in

Operations Research

mansans

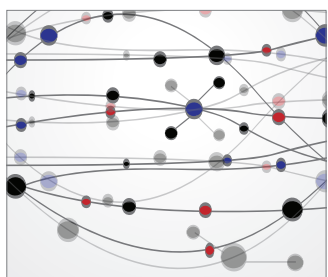

The Scientific World Journal
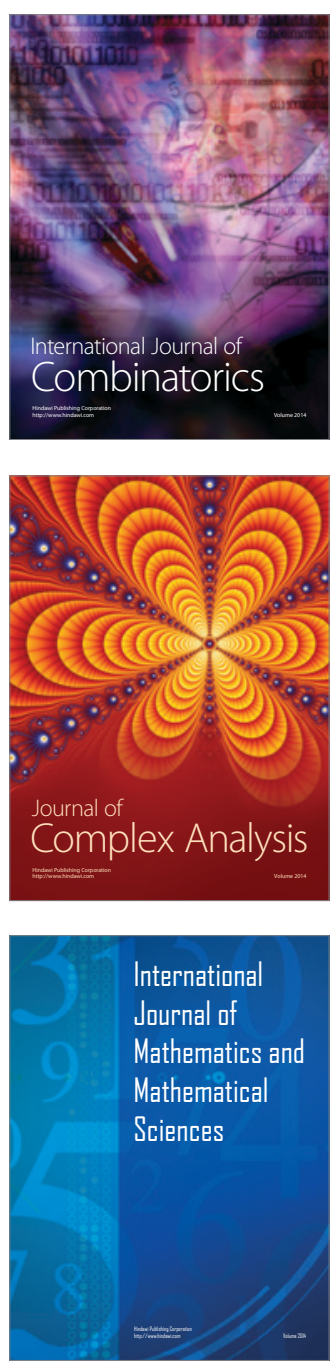
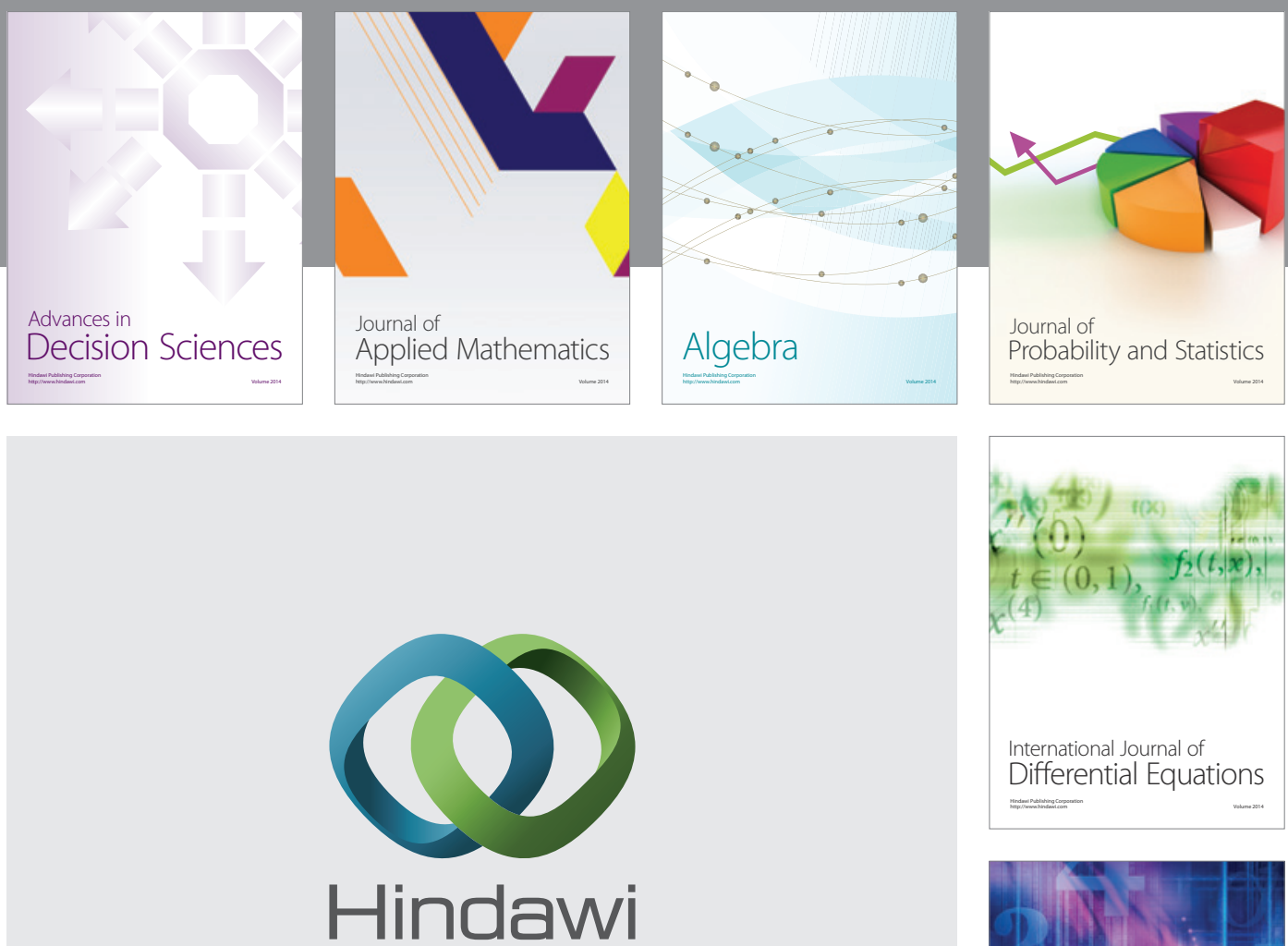

Submit your manuscripts at http://www.hindawi.com
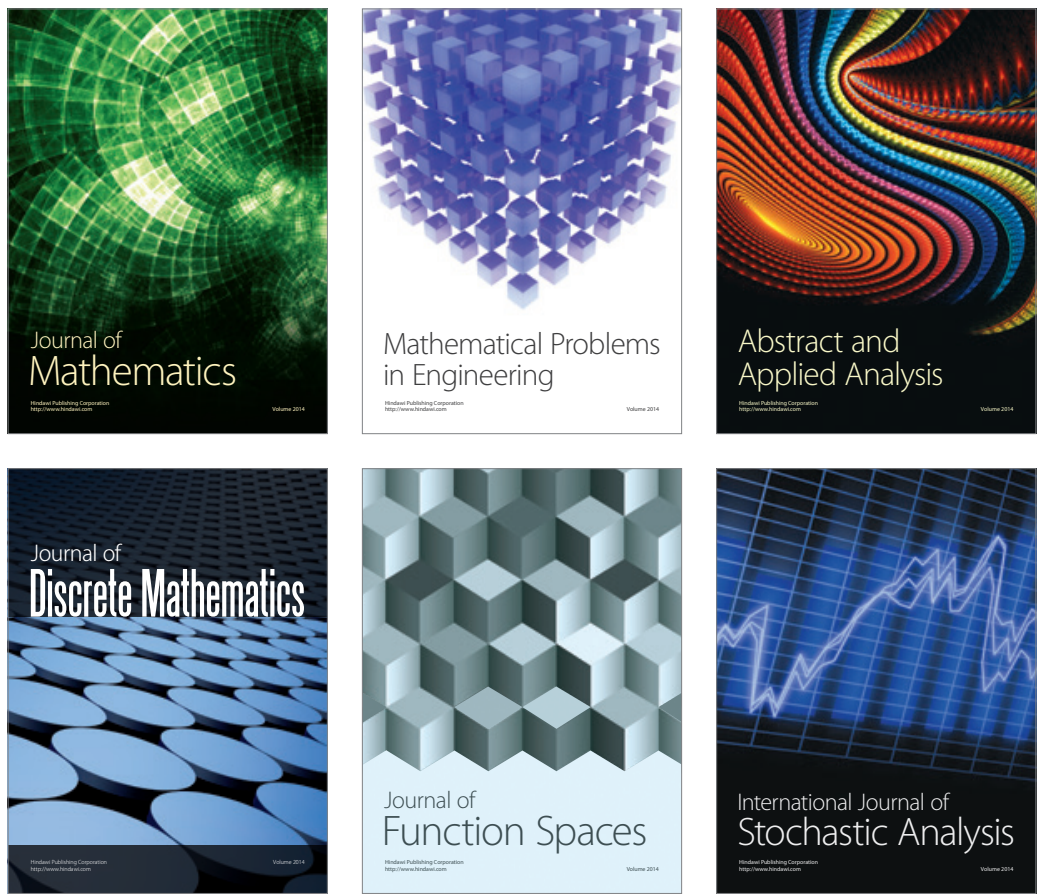

Journal of

Function Spaces

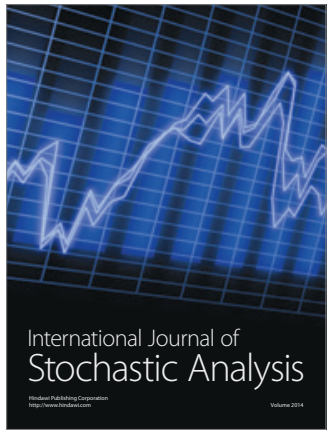

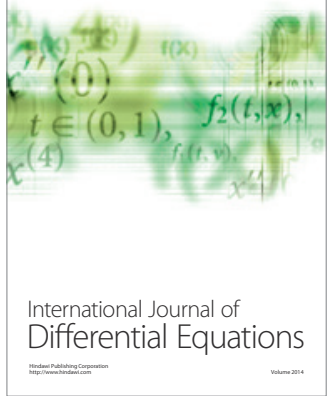
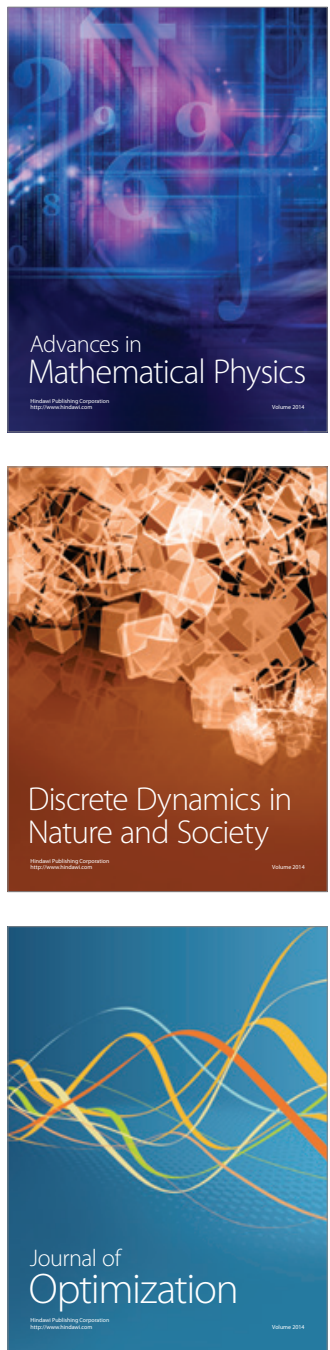